\title{
EXPERIÊNCIA COM APLICAÇÃO DE TOXINA BOTULÍNICA A EM 115 PACIENTES
}

\author{
LUIZ AUGUSTOF. ANDRADE*, VANDERCI BORGES**, \\ HENRIQUE BALLALAI FERRAZ***, SONIA MARIA AZEVEDO-SILVA ****
}

\begin{abstract}
RESUMO - A toxina botulínica A é a forma mais eficaz de tratar algumas distonias focais e o espasmo hemifacial. Devido a seu custo ainda elevado no nosso país, seu uso ainda não é disseminado. Mostramos nossa experiência com a toxina botulínica $\mathrm{A}$ em 115 pacientes, sendo 45 com espasmo hemifacial que apresentaram melhora acentuada ou total em $100 \%$. Em 20 pacientes com blefaroespasmo essencial houve melhora acentuada ou total em 70\% . Na síndrome de Meige, representada por 14 pacientes, a melhora acentuada ou total foi observada em 71,4\% . Em 23 pacientes com distonia cervical observou-se melhora acentuada em 65,2\%. Distonia da mão, com 6 pacientes, apresentou o menor índice de melhora acentuada ( 2 pacientes, $33,3 \%$ ). As complicaç̃es de caráter transitório ocorreram, sendo as mais frequentes ptose palpebral e diminuição da força palpebral e a mais grave, pneumonia aspirativa. Acreditamos que a toxina é forma eficaz e habitualmente segura de tratamento destas condições clínicas.
\end{abstract}

PALAVRAS-CHAVE: toxina botulínica A, Botox, distonias focais, espasmo hemifacial.

\section{Botulinum toxin A: experience in the treatment of 115 patients}

ABSTRACT - Botulinum toxin A is the more efficient therapy of focal dystonias and hemifacial spasm. Our experience with botulinum toxin A injections in 115 patients is reported. Marked or total improvement was achieved in all 45 patients with hemifacial spasm, in $70 \%$ of 20 patients with essential blepharospasm and in $71.4 \%$ of 14 patients with Meige's syndrome. In $65.2 \%$ of 23 patients with cervical dystonia marked but no total improvement was obtained. The worse results were seen in the 6 patients with hand dystonia (writers cramp), in whom marked improvement was obtained in just two. Mild and transient complications occurred in up to 24.4\%, eyelid ptosis and eyelid weakness being the most frequent. One patient with Meige's syndrome had an aspiration pneumonia following dysphagia. Our results are in agreement with others, showing that botulinun toxin $A$ is a useful and safe treatment for these conditions.

KEY WORDS: botulinun toxin A, Botox, focal dystonias, hemifacial spasm.

A toxina botulínica $\mathrm{A}$ é considerada a mais potente toxina natural existente, atuando de maneira a bloquear a transmissāo neuromuscular de acetilcolina. Sua utilização clínica se iniciou na década de 80 pelo oftalmologista Allan Scott, na Califórnia (USA) ${ }^{15}$, para a correção de estrabismo. Após isso passou a ser utilizada em movimentos involuntários como o blefaroespasmo ${ }^{2}$ e espasmo hemifacial, tornando-se o tratamento de primeira escolha nestas condições; posteriormente passou a ser utilizada em distonia cervical, disfonia espástica, distonias focais das māos e pernas, distonia de açāo específica e tremores ${ }^{7}$. O espectro das indicaçōes de tratamento pela toxina botulínica aumentou consideravelmente nos últimos anos, passando a ser indicado em tratamento da espasticidade, em

Setor de Distúrbios do Movimento da Disciplina de Neurologia Clínica do Departamento de Neurologia e Neurocirurgia da Universidade Federal de São Paulo - Escola Paulista de Medicina. São Paulo: *Professor Livre Docente, Chefe da Disciplina de Neurologia Clínica; **Mestre em Neurologia; ***Doutor em Neurologia, Professor da Pós-Graduaçåo; ****Mestre em Neurologia. Aceite: 29-abril-1997. 
programas de reabilitação, em disfunção do esfincter da bexiga ou anal, experimentalmente em casos de cefaléia tensional e até como tratamento estético, eliminando rugas faciais.

Nosso objetivo é mostrar a experiência com aplicação da toxina botulínica $A$ em um grupo de pacientes desde outubro de 1991 até maio de 1996.

\section{MATERIAL E MÉTODOS}

Avaliamos 115 pacientes provenientes do ambulatório de neurologia no Setor de Distúrbios do Movimento da Disciplina de Neurologia Clínica da Universidade Federal de Sảo Paulo - Escola Paulista de Medicina. Eram 45 pacientes com espasmo hemifacial, $24 \mathrm{com}$ blefaroespasmo, $14 \mathrm{com}$ síndrome de Meige, 26 com distonia cervical e 6 com distonia da mão, na forma de distonias de ação específica.

Estudamos a distribuiçāo quanto ao sexo, idade, a dose de toxina aplicada, o número total de aplicações, a duraçāo média do tempo do efeito, os resultados e as complicações. A melhora foi considerada discreta quando menor que $50 \%$, acentuada quando maior que $50 \%$ e total, $100 \%$, de acordo com a informaçăo obtida pelo paciente e objetivamente ao exame. A avaliação do resultado foi realizada após $\mathbf{4}$ semanas da aplicação.

Os pacientes com distonia da mão foram submetidos à eletroneuromiografia antes da aplicação.

A toxina utilizada foi a americana (Botox, Laboratório Allergan) com $100 \mathrm{U}$ de toxina liofilizada em cada frasco, com diluições em 1 ou $2 \mathrm{ml}$ de solução fisiologica a $0,9 \%$, correspondendo respectivamente a $0,1 \mathrm{ml}$ $=10 \mathrm{U}$ e $0,1 \mathrm{ml}=5 \mathrm{U}$.

\section{RESULTADOS}

A Tabela 1. mostra a distribuição da amostra de acordo com a condição clínica. Os resultados obtidos podem ser vistos na Tabela 2. Quatro pacientes com blefaroespasmo e 3 com distonia cervical não aparecem nesta tabela pelo fato de nāo terem retornado à consulta até o encerramento da coleta dos resultados.

Os 3 pacientes com distonia cervical que não obtiveram melhora eram pacientes com distonia generalizada e apresentavam história com mais de 5 anos de início. Dois pacientes com síndrome de Meige apresentavam apraxia de abertura ocular além do blefaroespasmo, o que concorreu para que não houvesse qualquer melhora. Obviamente, com 259 aplicaçōes realizadas em 115 pacientes, houve a oportunidade de aplicar repetidamente a toxina em uma parte dos pacientes e apenas uma vez em outra parte. Neste estudo não nos dedicamos a comparar os grupos submetidos a apenas uma injeção com os que receberam injeçōes repetidas. Além do mais, os pacientes que foram injetados mais de uma vez, de modo geral, obtiveram o mesmo nível de melhora que na(s) anterior(es), mostrando não haver perda da eficácia com as repetiçōes.

Tabela 1. Distribuição da amostra de acordo com a condição clínica.

\begin{tabular}{|c|c|c|c|c|c|}
\hline & $\begin{array}{c}\text { Espasmo } \\
\text { hemifacial }\end{array}$ & $\begin{array}{l}\text { Blefaro- } \\
\text { espamo }\end{array}$ & $\begin{array}{l}\text { Síndrome de } \\
\text { Meige }\end{array}$ & $\begin{array}{l}\text { Distonia } \\
\text { cervical }\end{array}$ & $\begin{array}{l}\text { Distonia } \\
\text { da mão }\end{array}$ \\
\hline $\mathrm{n}$ total pacientes & 45 & 24 & 14 & 26 & 6 \\
\hline Masc / Fem & $15 / 30$ & $10 / 14$ & $1 / 13$ & $13 / 13$ & $4 / 2$ \\
\hline Idade média-anos (variação) & $53(31-79)$ & $60(39-79)$ & $59,7(46-68)$ & $40,5(21-67)$ & $40,1(26-54)$ \\
\hline Dose média-U (variação) & $34,5(25-42,5)$ & $57(40-70)$ & $65,8(50-100)$ & $254,7(150-350)$ & $81,6(36-150)$ \\
\hline $\begin{array}{l}\text { t médio efeito (meses) } \\
\text { (variação) }\end{array}$ & $3,4(1-8)$ & $2,9(0,5-7)$ & $2,5(2-3)$ & $4,2(1-12)$ & $2(1-3)$ \\
\hline n total de aplicaçðes & 121 & 55 & 32 & 41 & 10 \\
\hline
\end{tabular}


Tabela 2. Resultados obtidos.

\begin{tabular}{cccccc}
\hline Melhora & Espasmo facial & Blefaroespasmo & Síndrome de Meige & Distonia cervical & Distonia da måo \\
\hline Total & $9(20,0 \%)$ & $3(15 \%)$ & $2(14,3 \%)$ & & \\
Acentuada & $36(80,0 \%)$ & $11(55 \%)$ & $8(57,1 \%)$ & $15(65,2 \%)$ & $2(33,3 \%)$ \\
Discreta & & $3(15 \%)$ & $1(7,1 \%)$ & $5(21,7 \%)$ & \\
S / melhora & & $3(15 \%)$ & $3(21,4 \%)$ & $3(13 \%)$ & $4(66,7 \%)$ \\
Total & $45(100 \%)$ & $20(100 \%)$ & $14(100 \%)$ & $23(100 \%)$ & $6(100 \%)$ \\
\hline
\end{tabular}

As complicações mais frequentemente encontradas foram:

a) Espasmo facial: diminuição da força palpebral em $11(24,4 \%)$ pacientes, ptose palpebral em $6(13,3 \%)$, apagamento da rima labial em $2(4,4 \%)$, paralisia facial em $2(4,4 \%)$, fraqueza da boca em $5(11,1 \%)$ e diplopia em $1(2,2 \%)$;

b) Blefaroespasmo: ptose palpebral em $8(40 \%)$, diminuição da força palpebral em $1(5 \%)$, edema palpebral em 2 (10\%), diplopia em 1 (5\%) e ardor ocular em 1 (5\%);

c) Síndrome de Meige: edema palpebral em $1(7,1 \%)$, ptose em $1(7,1 \%)$, disfagia em 1 $(7,1 \%)$, fraqueza da boca em $1(7,1 \%)$ e pneumonia aspirativa em $1(7,1 \%)$;

d) Distonia cervical: disfagia em 3 (13\%), dor cervical em 2 (8,7\%), fraqueza no pescoço em $1(4,3 \%)$;

e) Distonia da mão: fraqueza em dedos em $4(66,7 \%)$.

Estes resultados foram computados sem os 7 pacientes que não haviam retornado após as aplicações.

\section{DISCUSSÃO}

Todos os $\mathbf{4 5}$ pacientes com espasmo hemifacial apresentaram uma melhora acentuada (mais de 50\%) ou total. Na literatura é descrita melhora que varia de 82 a 100\% 3.8 .14 . As complicaçōes mais frequentes e de caráter transitório foram diminuição da força palpebral, ptose palpebral e fraqueza da musculatura peri-bucal, algumas vezes com aspecto de paralisia facial periférica. Uma das pacientes com espasmo facial, que apresentou fraqueza na boca na primeira aplicação, solicitou que só fosse injetado o músculo orbicular do olho na segunda sessão. Mesmo assim apresentou apagamento da rima labial homolateral. Isto poderia ser usado como argumento da existência de uma açăo da toxina à distância do ponto injetado. Este efeito distante já foi discutido por Lange e col. ${ }^{12}$, num estudo duplo cego que mostra alterações eletrofisiológicas no membro superior, através da eletroneuromiografia de fibra única, em pacientes com torcicolo espasmódico, entretanto sem evidência clínica de fraqueza muscular.

Estudos eletrofisiologicos demonstram transmissão "efáptica", que é a transmissão de um potencial de açāo através de uma sinapse eletrônica de um axônio ao vizinho, sem ser mediada por neurotransmissor. No espasmo hemifacial, isto é demonstrado pela resposta em um músculo facial à estimulação de um ramo do sétimo nervo que não inerva o músculo, e que é tardia com respeito à resposta direta no músculo que é inervado. Por exemplo, no estímulo do ramo zigomático observase resposta imediata no músculo orbicular do olho e tardia no ramo mandibular e vice versa ${ }^{3}$. Com o uso da toxina botulínica, Geller e col. ${ }^{3}$, observaram que não houve alteração da transmissão excitatória do ramo zigomático ao mandibular e vice versa, mas a amplitude de resposta estava diminuída. Por outro lado Glocker e col. ${ }^{4}$ observaram redução do potencial de ação muscular de $40 \%$ no músculo orbicular injetado. Năo houve alteração da resposta tardia do zigomático ao ramo mandibular. Entretanto, nāo conseguiram obter resposta do músculo do mento à estimulação do ramo orbicular. Os autores acreditam que isto pode ser explicado pela captação preferencial da toxina botulínica $A$ 
por sinapses hiperativas envolvidas na transmissão "efâptica". Uma ação decorrente de difusão da toxina a partir do local injetado, no entanto, é pouco provável. Quase sempre se pode demonstrar, por trás dos movimentos clônicos ou tônicos da hemiface, uma fraqueza muscular subjacente, com hipotonia muscular, o que é diferente dos casos de distonia facial, em que existe aumento do tono muscular e também da contratilidade muscular. Esta fraqueza subjacente poderia concorrer para maior susceptibilidade local, por qualquer dos mecanismos que a toxina possa estar agindo.

A duração média do efeito foi de 3,4 meses (em torno de 14 semanas) e um dos pacientes mostrou efeito de 8 meses. Doses maiores tendem a produzir efeitos mais duradouros, porém com efeitos colaterais de fraqueza muscular mais acentuados. Mauriello e Aljian ${ }^{13}$ mostram duração do efeito em torno de 16 semanas para $50 \%$ de sua amostra.

No grupo de pacientes com blefaroespasmo essencial a melhora foi acentuada ou total em $70 \%$ dos pacientes e discreta em $15 \%$, não tendo ocorrido qualquer melhora em 3 pacientes (15\%). A duração média do efeito foi de 2,9 meses (em torno de 12 semanas). Jankovic e col. ${ }^{10}$, obtiveram melhora em $94 \%$ dos pacientes, com média de duração de 15,7 semanas. Três dos nossos pacientes não apresentaram qualquer melhora, sendo que $2 \mathrm{em}$ duas sessōes. A existência de componente de apraxia da abertura dos olhos mesclado ao blefaroespasmo, pode ser lembrada nestes casos. As complicaçōes mais frequentes foram ptose palpebral em $8(40 \%)$, sendo bilateral em $2(10 \%)$ e edema palpebral em $2(10 \%)$. A ptose palpebral tem sido descrita como uma das complicações mais frequentes, ocorrendo em torno de $10 \%{ }^{6}$. Acreditamos que, em nossa série, as causas para maior número de ocorrências de ptose palpebral possam ser falhas técnicas, com doses acima do desejável aplicadas nos cantos internos e externos da pálpebras superiores. Injetando-se doses de 2,5 U, ao invés de $5 \mathrm{U}$ nestes pontos, passamos a ter menor ocorrência de ptose palpebral.

Na síndrome de Meige, melhora acentuada ou total ocorreu em 10 pacientes $(71,5 \%)$; em 3 pacientes não houve qualquer resposta. Dois destes apresentavam apraxia de abertura ocular. A apraxia de abertura ocular pode ocorrer associada à doença de Parkinson, à paralisia supranuclear progressiva (PSP - síndrome de Steele, Richardson, Olszewski), outras enfermidades extrapiramidais ou mesmo isoladamente. Frequentemente é confundida com o blefaroespasmo essencial. $O$ fenômeno diferencial é que no blefaroespasmo existe sempre contração muscular que mantém forçadamente os olhos fechados e na apraxia de abertura das pálpebras o paciente fecha os olhos e não mais consegue abri-los, porém sem contração muscular. Apenas o mecanismo de inibiçāo reflexa entre o elevador da pálpebra e o orbicular do olho se encontra alterado. Na apraxia de abertura, termo que nem todos aceitam facilmente, também não ocorre o piscamento repetido que é tão comum no blefaroespasmo. $O$ fator complicador é que uma proporção de pacientes com o blefaroespasmo essencial apresentam também algum grau de apraxia de abertura das pálpebras, de modo que o resultado da aplicaçāo, fazendo-se predominantemente sobre a contração muscular excessiva, pode não ser satisfatório. Esta associação também ocorre nos pacientes com a síndrome de Meige, como relatado anteriormente. Existem alguns relatos na literatura de melhora da apraxia com toxina botulínica $A^{5.11}$. Já tivemos pacientes com apraxia de abertura dos olhos que responderam bem às aplicações de toxina botulínica. A explicação para esta resposta não parece muito fácil.

Os efeitos colaterais ocorreram em $5(35,7 \%)$ com ptose palpebral, diplopia, edema palpebral, e o caso mais grave foi de uma paciente que apresentou disfagia seguida de pneumonia aspirativa, necessitando internação em terapia intensiva, porém, felizmente, recuperando-se. Havia recebido injeçōes também na musculatura cervical (cuticular do pescoço) e músculos submandibulares.

Na distonia cervical, obtivemos melhora acentuada em $15(65,2 \%)$ e discreta em $5(21,2 \%)$, com duração média do efeito de 4,2 meses (em torno de 18 semanas). Neste grupo não tivemos pacientes com melhora completa, total. Os 3 pacientes que não apresentaram melhora eram portadores de distonia generalizada com mais de 5 anos de história. Tem sido descrita melhora, que varia de 61 a 90\%, da posição da cabeça ${ }^{1,5,10}$ e melhora dramática na dor cervical. Nossos pacientes também confirmaram melhora marcada das dores musculares cervicais e dorsais. Os efeitos colaterais mais encontrados foram disfagia em 3 (13\%), dor cervical em $2(8,7 \%)$ e fraqueza no pescoço em $1(4,3 \%)$. Blackie e Lees' observaram disfagia em $28 \%$ de sua amostra e duração média do efeito de 12 semanas. 
Entre as causas para as falhas de resposta devemos incluir dose inadequada, escolha errônea dos melhores músculos a serem injetados e o desenvolvimento de anticorpos. Esta última não deve ser levada muito em consideração em nossos pacientes pois houve número pequeno de repetições, o que costuma ser fator importante no desenvolvimento de anticorpos, além do tamanho das doses. Num estudo longitudinal com toxina botulínica A em pacientes com blefaroespasmo e distonia cervical, Jankovic e Schwartz ${ }^{8}$ observaram que 4,3 \% dos pacientes com distonia cervical não responsivos mostraram a presença de anticorpos e posteriormente os mesmos autores ${ }^{y}$ testaram 86 pacientes para a presença de anticorpos, sendo que 20 eram positivos e estes não apresentavam resposta em pelo menos duas sessōes consecutivas. Greene e col. ${ }^{5}$ estimam em 7 a $10 \%$ a prevalência de sua população de distonia cervical com a presença de anticorpos. Nestes casos uma boa opção seria o uso da toxina botulínica $\mathrm{F}^{8,16}$.

Na distonia da mão observamos melhora em $2(33,3 \%)$ pacientes e 4 apresentaram fraqueza dos dedos. Poungvarin e col. ${ }^{14}$ utilizaram toxina em 92 pacientes com distonia ocupacional com melhora em 67,3\%. Jankovic e col. ${ }^{10}$ relataram melhora moderada em $77 \%$ em sua série de 22 pacientes, acompanhada de fraqueza transitória dos dedos.

Nos pacientes com distonia da mão há a dificuldade em localizar os músculos responsáveis pela disfunção, pois na maioria dos casos se situam profundamente e não são fáceis de serem identificados. É necessário o uso da eletroneuromiografia. Mesmo com o uso desta, não conseguimos bons resultados. A complexidade dos músculos das mãos e antebraços envolvidos nas distonias ocupacionais, como a cāimbra do escrivão, dos digitadores de computador, dos músicos ou esportistas, concorre com a dificuldade de se abordar os músculos adequados. É uma área onde ainda se aguarda por melhores indicadores técnicos para as injeções.

Nós concluímos que a aplicação de toxina botulínica A é forma eficaz e habitualmente segura de tratamento do espasmo hemifacial e distonias focais, com resultados superiores aos que se consegue com os variados medicamentos. Todas as complicaçōes foram transitórias $\mathrm{e}$, com excessāo de um caso de penumonia aspirativa, foram benignas.

\section{REFERÊNCIAS}

1. Blackie JD, Lees AJ. Botulinum toxin treatment in spasmodic torticollis. J Neurol Neurosurg Psychiatry 1990;53:640-643.

2 Frueh B, Felt D, Wojno T, Musch D. Treatment of blepharospasm with botulinum toxin. Arch Ophthalmol 1984;102:1464-1468.

3. Geller BD, Hallet M, Ravits J. Botulinum toxin therapy in hemifacial spasm; clinical and electrophysiologic studies. Muscle Nerve 1989;12:716-722.

4. Glocker FX, Guschlbauer B, Licking CH, Deuschl G. Effects of local injections of botulinum toxin on electrophysiological parameters in patients with hemifacial spasm: role of synaptic activity and size of motor units. Neurosci Lett 1985;187:161-164.

5. Greene P, Kang U, Fahn S, Brin M, Moskowitz C, Flaster E. Double-blind, placebo-controlled trial of botulinum toxin injections for the treatment of spasmodic torticollis. Neurology 1990;40:1213-1218.

6. Greene P, Fahn S, Brin M, Blitzer A. Botulin toxin therapy. In Marsden, C.D., Fahn, S. Movement Disorders 3. Oxford, Butterworth-Heinemann, 1994:477-502.

7. Jankovic J, Brin MF. Therapeutic uses of botulinum toxin. N Engl J Med 1991;324:1186-1194.

8. Jankovic J, Schwartz KS. Longitudinal experience with botulinum toxin injections for treatment of blepharospasm and cervical dystonia. Neurology 1993;43:834-836.

9. Jankovic J, Schwartz KS. Response and immunoresistance to botulinum toxin injections. Neurology 1995;45:1743-1746.

10. Jankovic J, Schwartz KS, Donovan DT. Botulinum toxin treatment of cranial-cervical dystonia, spasmodic dysphonia, other focal dystonias and hemifacial spasm. J Neurol Neurosurg Psychiatry 1990;53:633-639.

11. Katz B, Rosemberg JH. Botulinum therapy for apraxia of eyelid opening. Am J Ophthalm 1987;103:718-719.

12. Lange DJ, Rubin M, Green PE, Kang UJ, Moskowitz CB, Brin MF, Lovelace RE, Fahn S. Distant effects of locally injected botulinum toxin: a double-blind study of single fiber EMG changes. Muscle Nerve 1991;14:672-675.

13. Mauriello JA, Aljian J. Natural history of treatment of facial dyskinesias with botulinum toxin: a study of 50 consecutive patients over seven years. Br J Ophthalmol 1991;75:737-739.

14. Poungvarin N, Devahastin V, Viriyavejakul A. Treatment of various movement disorders with botulinum $A$ toxin injection: an experience of 900 patients. J Med Assoc Thai 1995;78:281-287.

15. Scott A. Botulinum toxin injection into extraocular muscles as an alternative to strabismus surgery. Ophthalmology 1980;87:1044-1049.

16. Sheean G, Lees $A J$. Botulinum toxin $F$ in the treatment of torticollis clinically resistant to botulinum toxin $A$. J Neurol Neurosurg Psychiatry 1995;59:601-607. 